\title{
Modelo de suprimento sanguíneo do intestino grosso do tamanduá bandeira (Myrmecophaga tridactyla)
}

\author{
Model of blood supply to the intestine of Giant anteater (Myrmecophaga tridactyla)
}

\author{
Ana Lúcia Rezende de Souza' ${ }^{I}$ Lorenna Cardoso Rezende ${ }^{I I}$ Amanda Rocha Mortoza ${ }^{I I I}$ \\ Jussara Rocha Ferreira ${ }^{\text {III* }}$
}

RESUMO

Em seis animais adultos Myrmecophaga tridactyla estudou-se o modelo de suprimento sanguíneo do intestino grosso, que é dependente das artérias mesentérica cranial (AMCr) e caudal (AMC). Os espécimes coletados conforme as normas do IBAMA (Instituto Brasileiro do Meio Ambiente e dos Recursos Naturais Renováveis) foram perfundidos com água $\left(40^{\circ} \mathrm{C}\right)$, injetados com látex corado, fixados em formol $e$ conservados em solução alcoólica (50\%). O mesocólon está disposto na linha sagital mediana e fixa o intestino grosso à parede dorsal do abdome. Derivaram da AMC: a artéria retal cranial, sete a 14 artérias cólicas e uma ou duas artérias ileocólicas, que apresentaram anastomoses de irrigação com a AMCr. A AMC finaliza-se na borda mesocólica das alças intestinais, emitindo ramos cólicos retos a partir das arcadas justacólicas, que penetram na intimidade da musculatura longitudinal. Ao longo do trajeto da AMC foram observadas ilhas arteriais, e a região ileocólica apresentou maior densidade vascular.

Palavras-chave: morfologia, mesentério, artérias, Myrmecophaga tridactyla, Xenarthra.

\section{ABSTRACT}

This research aimed to study the model of large intestine blood supply, which is dependent on the cranial mesenteric artery (AMCr) and caudal mesenteric artery (AMC), in six adults of Myrmecophaga tridactyla. The specimens were collected in accordance with the IBAMA standards (Brazilian Institute of the Environment) and were perfused with water $\left(40^{\circ} \mathrm{C}\right)$, injected with colored latex, fixed in formaldehyde and preserved in alcohol solution (50\%). The mesocolon is positioned in the median sagittal line and fixes the large intestine to the dorsal wall of the abdomen. The following is derived from the AMC: the cranial rectal artery, from 7 to 14 colic arteries and one or two ileocolic arteries, which present irrigation anastomosis with AMCr. The AMC finishes at the mesocolic edge of the bowel loop, generating straight colic branches from juxtacolic arcades, which penetrate the intimacy of longitudinal muscles. Arterial islands were observed along the AMC path and the ileocolic region showed higher vascular density.

Key words: morphology, mesentery, arteries, Myrmecophaga tridactyla, Xenarthra.

\section{INTRODUÇÃO}

Estudar o modelo de vascularização em Xenarthra, nesse caso, o intestino grosso do Myrmecophaga tridactyla, conhecido popularmente como tamanduá bandeira, faz parte de um ensaio que busca compreender nesses vertebrados, mais primitivos, alguns aspectos dos princípios de construção corporal.

Do ponto de vista da anatomia aplicada, os chamados segmentos orgânicos no intestino grosso e em outros órgãos apresentam territórios vasculares praticamente independentes, podendo vir a ser separados e removidos cirurgicamente ou ainda servir para reconstrução de vísceras (SIQUEIRA \& LÁZAROda-SILVA, 2003). O princípio da segmentação anátomocirúrgica (DIDIO, 1998) observa a subdivisão dos órgãos de acordo com a distribuição de seus vasos

'Universidade Federal de Goiás Campus Jataí (UFG), Jataí, GO, Brasil.

IUniversidade de São Paulo (USP), São Paulo, SP, Brasil.

IIIFaculdade de Medicina, Universidade de Brasília (UnB), 70735-000, Brasília, DF, Brasil. E-mail: jussararocha@unb.br. *Autor para correspondência. 
sanguíneos e linfáticos, nervos e ductos. Esse princípio tem aplicação prática em cirurgias de rececção visceral, conforme experimentos realizados no intestino (STACCHINI et al., 1982), rins (GRAVES, 1954; CARVALHO et al., 2008) e baço (ZAPPALÁ, 1963).

Outro aspecto a ser considerado é o que diz respeito ao fato de que os desenhos anatômicos mais primitivos conservam os princípios de construção corporal com maior intensidade, mesmo após terem passado pelos processos filo e ontogenéticos dos quais derivam suas formas atuais. Ao observar o peritônio do tamanduá bandeira, dois aspectos chamam a atenção: o primeiro é a não aderência dos cólons à parede dorsal, conforme ocorre em outros vertebrados (SIQUEIRA \& LÁZARO-da-SILVA, 2003; MOORE \& PERSAUD, 2008; PÉREZ et al., 2008); e o segundo é a fixação dos intestinos grosso e delgado à mesma macromembrana, ou seja, uma única raiz serosa vertical prende cranialmente o mesentério e caudalmente o mesocólon. Embora a literatura faça referência sobre a evolução do mesentério (GRASSÉ, 1965), esta não esclarece que aspectos levaram esse animal a exibir esse modelo de peritônio e dos vasos que nele transitam. Como o modo de distribuição dos vasos sanguíneos é um dos indicativos do estágio evolutivo (BUGGE, 1974) do desenho anatômico atual, optou-se por analisar o suprimento sanguíneo do intestino grosso em tamanduá bandeira.

Com base no princípio da segmentação, que significa literalmente parte de órgãos, DIDIO (1998) definiu segmento como uma seção ou um território de um órgão ou de uma outra estrutura que possa ser limitada naturalmente ou artificialmente do restante do organismo. Nesse sentido, a partir da análise dos dados dessa pesquisa com base nas descrições clássicas (TESTUT \& LATARJET, 1960; LLORCA, 1952), foram tecidas considerações a respeito da origem, do trajeto e do destino dos vasos dos animais estudados. O objetivo deste estudo, do ponto de vista da anatomia aplicada, foi verificar se os segmentos de intestino analisados representam territórios anatomocirúrgicos independentes ou interdependentes.

\section{MATERIAL E MÉTODOS}

Nesta pesquisa foi descrito quantitativamente o modelo de vascularização do intestino grosso em seis espécimes adultos de Myrmecophaga tridactyla ( $\mathrm{n}=3$ machos e $\mathrm{n}=3$ fêmeas) atropelados em rodovias do Estado de Goiás e do Paraná, coletados na forma da lei. O método incluiu a confecção de relatório macroscópico dos animais; canulação da aorta abdominal e perfusão com água aquecida $\left(40^{\circ} \mathrm{C}\right)$; injeção de látex corado (Neoprene $450^{\circledR}$ e Sulvinil Corante 2350-0003 ${ }^{\circledR}$ ); fixação em solução de formol (10\%) e conservação em solução de Laskovisk. Para fins de sistematização dos vasos, as dissecações foram realizadas sob mesoscopia de luz (Lts ${ }^{\circledR}$-Mod. 3700) e foto-documentação com câmera digital (Nikon $\mathrm{D} 40^{\circledR}$ ). A análise dos dados considerou a origem das artérias, a trajetória dos vasos pelo peritônio (mesocólon e mesentério), o modo de terminação e a presença ou não de anastomoses entre as artérias mesentérica cranial (AMCr) e a mesentérica caudal (AMC), além da formação de ilhas arteriais entre os leitos arteriais. Os equipamentos e produtos químicos utilizados nesta pesquisa foram adquiridos por licitação pública da Fundação Universidade de Brasília. Para fins de denominação utilizou-se a Nomina Anatômica Veterinária (2005).

\section{RESULTADOS E DISCUSSÃO}

A irrigação do intestino grosso em tamanduá bandeira é dependente da AMCr e da AMC. Para facilitar o entendimento da origem, do trajeto e da terminação dos vasos neste animal, é preciso destacar alguns aspectos do peritônio. As AMCr e AMC, ao se destacarem da face ventral da aorta abdominal no retro peritônio, alcançam a raiz do meso definitivo por meio do mesotélio, insinuando-se entre as duas lâminas do peritônio e deslocando-se em direção às alças intestinais. O mesocólon e o mesentério unidos formam uma macromembrana bilaminar, que sustenta espacialmente os vasos em posição anatômica, permitindo as subdivisões destes.

As AMCr e AMC são paralelas neste animal, que exibe um modelo primitivo de intestino grosso conforme GRASSÉ (1965). Após o desaparecimento do pedículo vitelínico, o intestino grosso não coalesce na parede dorsolateral do abdome (Figura 1A), como ocorre em outros mamíferos (LAT SHAW, 1987; MOORE \& PERSAUD, 2008). Portanto, as alças do intestino grosso, exceto o reto, são livres (Figura 1B). O intestino grosso é desprovido de cintas musculares longitudinais, com alças de configuração retilínea e calibre maior que o intestino delgado, e a musculatura longitudinal apresenta-se expressiva macroscopicamente (Figura 1C).

Estão presentes primitivas saculações e um pequeno ceco situado no hipocôndrio direito. A primeira alça de cólon inclina-se para a esquerda, assumindo, em relação ao ângulo duodeno jejunal, uma flexura descendente (cólica direita) após o que denomina-se cólon descendente. Esta corre crâniocaudalmente presa à parede dorsal pelo mesocólon, 


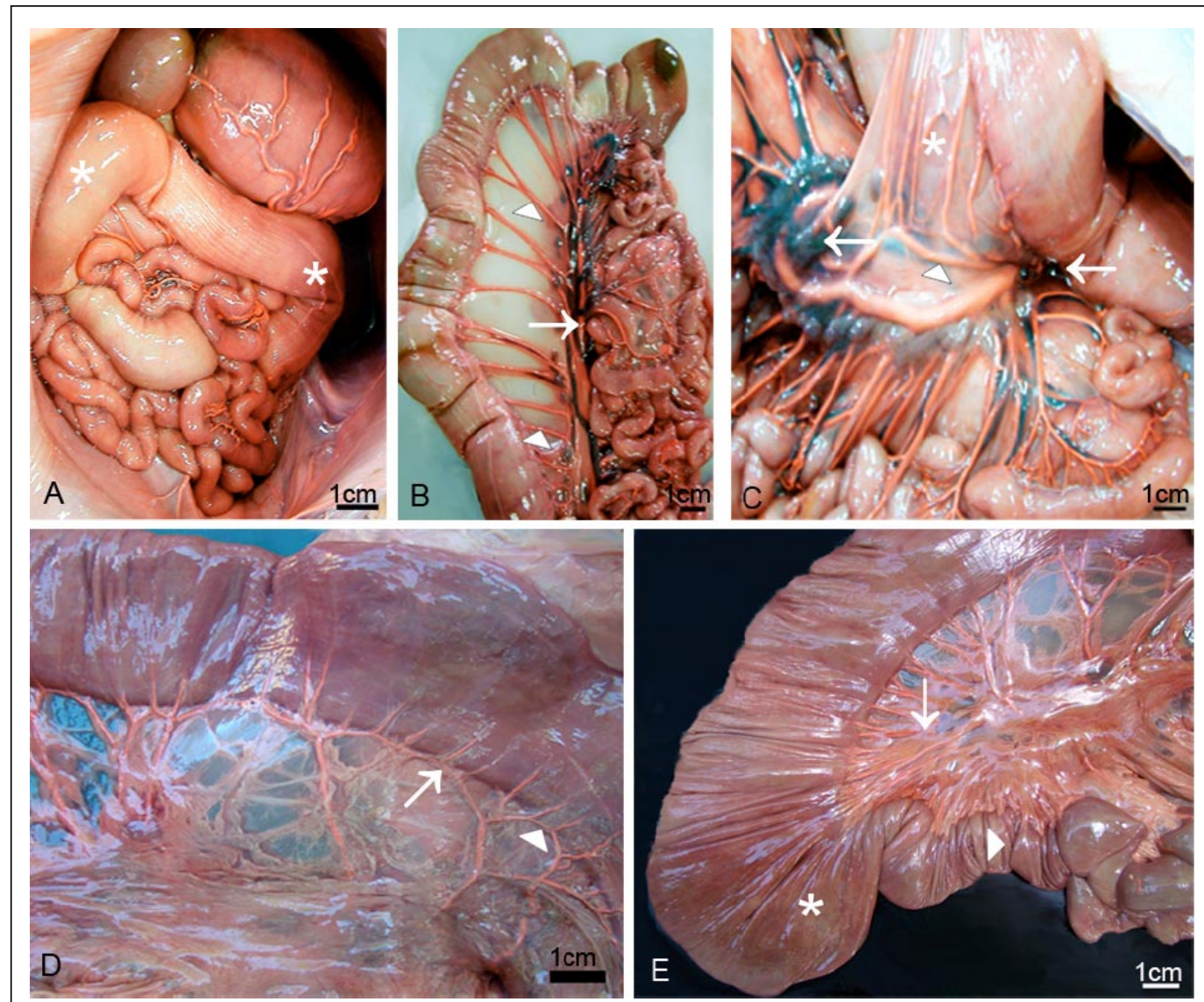

Figura 1 - Fotografia de aspectos da vascularização do intestino grosso de tamanduá bandeira. Destaca-se: em A, topografia geral das vísceras, detalhe das flexuras cólicas (*), com ausência de coalescência do intestino grosso à parede; em $\mathrm{B}$, topografia da $\mathrm{AMC}(\rightarrow)$, os ramos primários retos da AMC ( $)$; em C, a AMC $(\downarrow)$, linfonodos regionais $(\rightarrow)$ e detalhes do meso reto $(*)$; em $\mathrm{D}$, artéria arqueada de primeira ordem $(\rightarrow)$ e de segunda ordem $(\bullet)$; em E, as artérias retas na zona de transição íleo-cólica $(\rightarrow)$, o ceco $\left(^{*}\right)$ e o íleo ( $)$.

até adentrar na pelve maior como intestino reto (Figura 1A). No vazio pélvico, o peritônio visceral que envolve o intestino reto reflete-se como peritônio parietal. O intestino reto posiciona-se dorsocaudalmente no interior da pelve menor, alcançando o períneo, onde termina.

AAMC vasculariza todo o intestino grosso e os dois terços craniais do intestino reto (Figura 1B, $1 \mathrm{D}, 1 \mathrm{E})$. Exibe um comprimento de $20,5 \mathrm{~cm}$, corre paralelamente às alças retais e cólicas, estando de 3 a $4 \mathrm{~cm}$ afastada dessas alças (Figura 1D). A partir da raiz do mesocólon, os ramos colaterais da AMC irrigam sequencialmente: o intestino reto; o cólon ascendente; o cólon transverso e o ceco. O tronco principal da AMC, na medida em que percorre o interior da macromenbrana peritonial, tem independência em seu território de irrigação, exceto nos locais de anastomoses com a AMCr. AAMC emite ramos colaterais primários retos, cuja hierarquia de calibre diminui gradativamente ao longo da emissão destes. Desses vasos retos, derivamse artérias cólicas arqueadas. Essas artérias, ao se aproximarem da borda mesocólica, anastomosam-se, lateralmente, com o vaso paralelo. Foram observadas arqueações de primeira e de segunda ordem. Dos arcos justacólicos partem os ramos terminais retos nutridores da alça intestinal cujos microvasos irrigam, tanto o mesocólon, como os grupamentos linfonodulares regionais justaintestinais (Figura 1D).

O primeiro ramo colateral primário da AMC dirige-se ao intestino reto. Em um espécime, derivouse desta um ramo ascendente para o cólon descendente. Foram encontradas de sete a 13 artérias primárias retas destinadas às alças cólicas e de um a três ileocólicas (Tabela 1). Estas se subdividiram no mesocólon em ramos colaterais secundários e terciários. Suas terminações formam arcos justacólicos, 
Tabela 1 - Origem da artéria mesentérica caudal (AMC), quantidade de ramos colaterais primários destinados ao intestino grosso e registro da presença de anastomoses com a artéria mesentérica cranial (AMCr) em tamanduá bandeira, em função do número de animais e do sexo. Artéria renal esquerda (are).

\begin{tabular}{|c|c|c|c|c|c|c|c|}
\hline \multirow[b]{2}{*}{ Animal } & \multirow[b]{2}{*}{ Sexo } & \multirow[b]{2}{*}{ Origem } & \multicolumn{3}{|c|}{---------------'Ramos primários da AMC---------------- } & \multicolumn{2}{|c|}{------------Anastomoses------------ } \\
\hline & & & Intestino reto & $\begin{array}{c}\text { Cólon } \\
\text { descendente }\end{array}$ & Cólon transverso & Interanastomoses & AMCxAMCr \\
\hline 1 & $\mathrm{~F}$ & are & 1 & 10 & 2 & 1 & 2 \\
\hline 2 & $\mathrm{~F}$ & aorta & 1 & 9 & 1 & 5 & 2 \\
\hline 3 & M & aorta & 1 & 6 & 2 & 3 & 2 \\
\hline 4 & $\mathrm{~F}$ & aorta & 1 & 11 & 3 & 3 & 7 \\
\hline 5 & M & aorta & 1 & 6 & 1 & 3 & 3 \\
\hline 6 & M & aorta & 1 & 7 & 1 & 1 & 1 \\
\hline Total & & & 6 & 49 & 10 & 16 & 17 \\
\hline
\end{tabular}

Fonte: Dados de pesquisa de SOUZA et al. (2009).

representados pelas anastomoses entre os ramos arteriais paralelos: as artérias paracólicas. Destas originam-se pequenos vasos, em pares, na borda mesocólica da alça intestinal, cujas terminações atingem as duas faces do cólon, onde os vasos penetram na musculatura e se capilarizam.

Vale destacar que o cólon descendente recebe menor número de vasos primários, embora estes sejam de calibre relativamente maior. Foram detectadas interanastomoses entre as artérias cólicas, além de anastomoses entre os leitos da AMCr e AMC (Tabela 1).

Na região cranial do mesocólon, em local próximo à junção ileocecal, as artérias cólicas de segunda e terceira ordem aumentam de densidade e se subdividem próximas à alça intestinal, como ramos cólicos retos. Anastomoses de irrigação entre a AMCr e a AMC são constantes nesse setor, embora se façam presentes ao longo de todo o território de domínio das duas artérias (Tabela 1 e Figura 1E). A AMC não contribui isoladamente com a irrigação do cólon transverso e não vasculariza o ceco, sendo esse setor irrigado exclusivamente por ramos caudais da $\mathrm{AMCr}$ (Tabela 1).

A literatura sustenta que o tubo digestório dos embriões dispõe-se na linha sagital mediana abdominal, a partir da qual os mesentérios primitivos, dorsal e ventral, passarão por sequências de rotações e processos ontogenéticos de diferenciação, formando o tubo digestório no adulto (NODEN \& LAHUNTA, 1985; LAT SHAW, 1987). O intestino delgado derivase do intestino médio, sendo nutrido pela AMCr (LLORCA, 1952; SCHWARZE \& SCHÖDER, 1970; NODEN \& LAHUNTA, 1985; MOORE \& PERSAUD, 2008). O intestino grosso é ontogeneticamente derivado do intestino posterior, portanto vascularizado pela AMC. Nesse animal, a inserção definitiva do meso não se deslocou da linha sagital mediana (Figura 1B), conferindo à serosa abdominal um desenho muito característico da superordem Xenarthra (GARDNER, 2007).

Comparando-se o modelo de vascularização do intestino grosso do tamanduá bandeira com ungulados (SCHWARZE \& SCHÖDER, 1970; NICKEL et al., 1977; PÉREZ et al., 2008; HOFMANN, 2000; CLAUS et al., 2005) e roedores (CARVALHO et al., 1999), cabe destacar que o modelo do peritônio do tamanduá interfere parcialmente no arranjo do padrão arterial do intestino grosso, ou seja, o meso une o órgão à parede dorsal do corpo, conduzindo vasos e nervos. Conforme o entendimento de MOORE \& PERSAUD (2008), LLORCA (1952), LAT SHAW (1987), NODEN \& LAHUNTA (1985) e JUNQUEIRA \& ZAGO (1977), o peritônio é o caminho natural do vaso. No presente estudo, ficou claro que os mesos do tamanduá bandeira derivados do mesentério dorsal permaneceram no indivíduo adulto com características primitivas, não sofrendo rotação no eixo sagital mediano, do qual brotam as primitivas AMC e AMCr. No animal adulto, estas são semelhantes àquela nutridora da alça intestinal média, que se insinuou no pedículo vitelino e àquela que irriga a primitiva alça do intestino posterior (TESTUT \& LATARJET, 1960; JUNQUEIRA \& ZAGO, 1977; NODEN \& LAHUNTA, 1985). As AMCr e AMC correm paralelas entre si, mesmo após a diferenciação do intestino delgado, permanecendo fixadas na borda cranial do mesentério. As artérias mesentéricas e mesocólicas formam um leque arterial, cuja haste central representa o tronco principal das AMCr e AMC. Suas subdivisões primárias são seguimentares ou metaméricas, ou seja, é possível, mesmo no indivíduo adulto, reconhecer segmentos arteriais em série, supostamente no sentido dorso-ventral (DIDIO, 1998), 
o que pode ser indicativo do grau de maturidade evolutiva e que explicaria, em parte, o desenho do mesocólon não aderido à parede.

Nos mesos dos vertebrados superiores, perde-se essa característica (SCHWARZE \& SCHÖDER, 1970; NICKEL et al., 1977; GETTY, 1981; BARNWAL et al., 1982; EVANS, 1993; CLAUSS et al., 2005). Por outro lado, estudos recentes tratam da configuração dos vasos no mesentério e mesocólon em: quelônios (RODRIGUES et al., 2003), aves (PINTO et al., 1998), roedores (CARVALHO et al., 1999; MACHADO et al., 2000; MACHADO et al., 2002; MACHADO et al., 2006; CULAU et al., 2008) e ungulados (BARNWAL et al., 1982; PEDUTI NETO \& SANTIS, 1970; PEREIRA et al., 1978; MACHADO et al., 2000), entretanto, esses autores não detalharam o trajeto intraseroso dos vasos para que pudesse ser comparado.

A respeito dos cólons e do reto, PÉREZ et al. (2008) demonstraram a anatomia macroscópica do trato intestinal em Ozotoceros bezoarticus, detendose na descrição de vários aspectos destes, sem detalhar sua vascularização. Em humanos, SIQUEIRA \& LÁZARO-da-SILVA (2003) estudaram a artéria mesentérica inferior para a retirada do intestino reto. Esta esteve, em média, afastada da parede do cólon por $14,3 \mathrm{~cm}$, contra três a quatro centímetros no tamanduá bandeira. Cabe destacar uma grande diferença no modelo humano, neste a AMC é retroperitonial e no tamanduá bandeira esta situa-se entre os dois folhetos da serosa peritonial. A origem da AMC no retroperitônio, caudal à artéria renal, é fato coincidente com achados em outros animais: quelônios (RODRIGUES et al., 2003), aves (PINTO et al., 1998) e roedores (CARVALHO et al., 1999; MACHADO et al., 2006; CULAU et al., 2008).

Cabe destacar que em primatas, a emergência do vaso é lateral esquerda (SIQUEIRA \& LÁZARO da
SILVA, 2003), assim como em cães, bovinos, equinos e suínos (BRUNI \& ZIMMERL, 1947; NICKEL et al., 1977; GETTY, 1981; LAT SHAW, 1987; PEREZ et al., 2008). As alças intestinais nos vertebrados superiores passaram por extensas rotações, por isso o padrão arterial e a anatomia macroscópica do intestino e dos mesos se afastaram do modelo Xenarthra. GRASSÉ (1965) subdividiu o intestino pós-pilórico em móvel e posterior e argumenta que o último não se curva e nem se alonga, dependendo do hábito alimentar de certos mamíferos. Considerando que o tamanduá tem uma dieta insetívora, o intestino grosso manteve-se primitivo por estar adaptado às exigências funcionais. Possivelmente, isso favoreceu que as artérias conservassem esse padrão muito particular. DIZ et al. (2006) destacam que o Myrmecophaga tridactyla tem o intestino grosso de tamanho similar ao comprimento do corpo, o que também foi constatado neste trabalho (cerca de $32,6 \mathrm{~cm}$ em média), enquanto o Chaetophactus villosus, um Xenarthra onívoro, descrito pelo autor supracitado, apresenta o intestino mais curto (Tabela 2).

Naturalmente que as variações nos comprimentos dos mesos e dos intestinos interferem no modelo dos vasos, entretanto, no presente trabalho, não foram encontrados dados em animais próximos filogeneticamente, para fazer a comparação com os dados desse estudo. Embora a AMC tenha sido descrita no pato doméstico como ramo visceral caudal da aorta (PINTO et al., 1998) e o seu ramo cranial seja responsável por irrigar o intestino reto, dando uma pequena contribuição para os cólons, o modelo das aves é bem divergente dos mamíferos. Em roedores, cutias e nutria, a AMC bifurca-se em artéria cólica esquerda e retal cranial (CARVALHO et al., 1999; CULAU et al., 2008), aproximando-se dos ramos colaterais retos observados neste estudo. Não há alusões ao trajeto do vaso relacionando-o à

Tabela 2 - Comprimento, em centímetros, da artéria mesentérica caudal (AMC), do mesocólon e dos segmentos do intestino grosso, do ceco e dos cólons, descendente e transverso, de tamanduá bandeira em função do número de animais e do sexo.

\begin{tabular}{|c|c|c|c|c|c|c|c|}
\hline Animal & Sexo & AMC & Mesocólon & Сесо & $\begin{array}{c}\text { Cólon } \\
\text { descendente }\end{array}$ & $\begin{array}{l}\text { Cólon } \\
\text { transverso }\end{array}$ & Total \\
\hline 1 & F & 18,5 & 29,0 & 9 & 37,5 & 90,5 & 137,0 \\
\hline 2 & F & 22,5 & 38,0 & 8 & 48,0 & 102,0 & 158,0 \\
\hline 3 & M & 20,0 & 36,4 & 9 & 40,0 & 90,0 & 139,0 \\
\hline 4 & $\mathrm{~F}$ & 16,0 & 35,5 & 10 & 34,5 & 85,0 & 129,5 \\
\hline 5 & M & 21,5 & 40,5 & 12 & 36,0 & 55,0 & 103,0 \\
\hline 6 & M & 24,5 & 39,0 & 10 & 29,0 & 90,5 & 129,5 \\
\hline $\bar{X}$ & & 20,5 & 36,4 & 9,6 & 37,6 & 85,5 & 132,6 \\
\hline
\end{tabular}

Fonte: Dados de pesquisa de SOUZA et al. (2009). 
macromembrana peritonial, como foi constatado no tamanduá bandeira.

\section{CONCLUSÕES}

Conclui-se que os intestinos delgado e grosso fixam-se na parede dorsal da cavidade abdominal por uma grande prega peritonial. Essa prega de dupla face apresenta-se em forma de loja serosa, delgada, com pouquíssimo tecido conjuntivo. Denomina-se lâmina própria do mesentério, e essa lâmina é comum aos intestinos delgado e grosso. Sua borda cranial envolve as alças do intestino delgado e a caudal do intestino grosso. O intestino grosso do tamanduá bandeira é vascularizado por duas fontes de suprimento: as AMCr e AMC, que seguem na lâmina própria, também chamada de mesotélio peritonial. A AMCr supre o ceco e ocasionalmente o segmento inicial do cólon transverso. A AMC vasculariza o reto e os cólons.

A AMC é ramo ventral da aorta abdominal, cauldal à artéria renal esquerda. Em 100\% da amostra, os dois vasos (AMCr e AMC) correram paralelos na intimidade da serosa peritonial. No trajeto dos vasos, estes não somente irrigaram os segmentos intestinais, objeto deste estudo, mas também se prestaram como elementos de sustentação visceral. Os ramos colaterais da AMC apresentaram-se retilíneos, arqueados e terminais justaintestinais. Estes resultam da bifurcação dos ramos arteriais secundários ou terciários retos, que, a partir da borda mesocólica da alça intestinal, circundam-na para nutri-la. Foram encontradas interanastomoses entre os ramos retos primários e secundários da AMC. As artérias arqueadas justacólicas são intercomunicantes. AAMC estabelece anastomose, de irrigação, com a AMCr. Os cólons do tamanduá bandeira não exibem saculações e a musculatura longitudinal é difusa, formando alças relativamente retas.

\section{REFERÊNCIAS}

BARNWAL, A.K. et al. Anatomical and roentgenographic studies on the cranial mesenteric artery of buffalo. Haryana Veterinary, v.21, n.1, p.1-5, 1982.

BRUNI, A.C.; ZIMMERL, V. Anatomia degli animal domestic. Milano: Appiano Gentile, 1947. 2v, 763p.

BUGGE, J. The cephalic arterial system in insetivores, primates, rodents and lagomorphs, with special reference to the systematic classification. Acta Anatomica, v.87, p.1-160, 1974.

CARVALHO, M.A.M. et al. Artérias mesentéricas cranial e caudal em cutias (Dasyprocta agouti). Veterinária Notícias, v.5, n.2, p.17-24, 1999.
CARVALHO, M.A.M. et al. Segmentos anatomo-cirúrgicos arteriais do rim de cutias (Dasyprocta prymnolopha). Pesquisa Veterinária Brasileira, v.28, p.249-252, 2008. Disponível em: <http:// www.scielo.br/scielo.php?script=sci_arttext \&pid=S0100736X2008000500004\&nrm=iso>. Acesso em: 20 mai. 2009. ISSN: 0100-736X.

CLAUSS, M. et al. Observations on the macroscopic digestive anatomy of the Himalayan tahr (Hemitragus jemlahicus). Anatomia, Histologia, Embryologia, v.34, p.276-278, 2005. Disponível em: <http://dx.doi.org/10.1111/j.14390264.2005.00611.x>. Acesso em: 20 mai. 2009. ISSN: 14390264.

CULAU, P.O.V. et al. Ramos colaterais da artéria aorta abdominal em Myocastor coypus (nutria). Acta Scientiae Veterinarie, v.36, n.3, p.241-247, 2008. Disponível em: $<$ http://www.ufrgs.br/actavet/36-3/art792.pdf>. Acesso em: 20 mai. 2009. ISSN: 1679-9216 (Online).

DIDIO, L.J.A. Organologia. In: DIDIO, L.J.A. Tratado de anatomia aplicada. São Paulo: Póluss Editorial, 1998. 1v. Cap.6, p.91-110.

DIZ, M.J.O. et al. Registro de medidas y pesos del tubo digestivo de un ejemplar de Chaetophractus villosus. Edentata, v.7, n.5, p.23-25, 2006. Disponível em: <http://bioone.org/doi/abs/ 10.1896/1413-4411.7.1.23>. Acesso em: 20 mai. 2009. doi: 10.1896/1413-4411.7.1.23.

EVANS, H.E. The heart and the arteries. In: EVANS H.E. Miller's anatomy of the dog. 3.ed. Philadephia: Saunders, 1993. p.586-681.

GARDNER, A.L. Cohort placentalia owen, 1837. Magnorder Xenarthra Cope, 1889. In: ______. Mammals of South America, Marsupials, Xenarthrans, Shrews, and Bats. Chicago: University of Chicago, 2007. V.1, p.127-177.

GETTY, R. Anatomia dos animais domésticos. Rio de Janeiro: Interamericana, 1981. V.2, 1134p.

GRASSÉ, P. Traité de zoologie. Anatomie systématique, biologie vertébrés: genéralites embriologie topographique. Anatomie comparée. Paris: Masson et Cie Éditeurs, 1965. Tome XII, 1129p.

GRAVES, F.T. The anatomy of the intrarenal and its applications to segmental resection of the kidney. British Journal of Surgery, v.42, p.132-139, 1954.

HOFMANN, R.R. Functional and comparative digestive system anatomy of Arctic ungulates. Rangifer, v.20, p.71-81, 2000.

INTERNATIONAL COMMITTEE ON VETERINARY GROSS ANATOMICAL NOMENCLATURE. Nomina anatomica veterinaria. 5.ed. Hannover: Editorial Committee, 2005. $166 p$.

JUNQUEIRA, L.C.U.; ZAGO D. Fundamentos de embriologia humana. Rio de Janeiro: Guanabara Koogan, 1977. 275p.

LAT SHAW, W.K. Veterinary developmental anatomy: a clinically oriented approach. Missouri: Mosby, 1987. 283p. 
LLORCA, F.O. Intestino médio. In: Anatomia humana. Barcelona: Editorial Científico Médica, 1952. Tomo III, cap.10, p.459-528.

MACHADO, G.V. et al. Padrão de divisão e distribuição das artérias mesentéricas no ratão-do-banhado (Myocastor coypus - Rodentia: Mammalia). Biotemas, v.1, p.59-63, 2006. Disponível em: <http://www.biotemas.ufsc.br/volumes/pdf/ volume191/p59a63.pdf $>$. Acesso em: 30 mai. 2009. ISSN: 0103-1643.

MACHADO, G.V. et al. Suprimento arterial para as glândulas adrenais no ratão-do-banhado (Myocastor coypus Molina, 1782). Archives of Veterinary Science, v.7, n.2, p.9-14, 2002.

MACHADO, M.R.F. et al. Origem das artérias celíaca e mesentérica cranial em bubalinos (Bubalus bubalis, L. 1758). Brazilian Journal of Veterinary Research and Animal Science, v.37, p.99-104, 2000. Disponível em: <http:// scielo.br/scielo.php?script=sci_arttext \&pid=S141395962000000200002>. Acesso em: 30 mai. 2009. doi: $10.1590 / \mathrm{S} 1413-95962000000200002$.

MOORE, K.L.; PERSAUD T.V.N. O sistema digestório. In:____Embriologia clínica. 3.ed. Rio de Janeiro: Elsevier, 2008. p.214-244.

NICKEL, R. et al. The alimentary canal, general and comparative. In: ______. Anatomy of the domestic mammals. 2.ed. Hamburg: Verlog Paul Parey, 1977. p.99203.

NODEN, D.N.; LAHUNTA A. The embriology of domestic animals. Developmental mechanisms and malformations. Baltimore: Williams \& Wilkins, 1985. 367p.

PEDUTI, N.J.; SANTIS, P.I.L. Origem das artérias celíaca e mesentérica cranial, por tronco comum, em fetos de bovinos azebuados. Revista da Faculdade de Medicina Veterinária e Zootecnia da Universidade de São Paulo, v.15, n.1, p.1922, 1970 .

PEREIRA, J.G.L. et al. A origem das artérias celíaca e mesentérica cranial, por tronco comum, em carneiros da raça Corriedale. Revista da Faculdade de Medicina Veterinária e Zootecnia da Universidade de São Paulo, v.15, n.1, p.1922, 1978.

PÉREZ, W. et al. Observations on the macroscopic anatomy of the intestinal tract and its mesenteric folds in the pampas deer (Ozotoceros bezoarticus, Linnaeus 1758). Anatomia, Histologia, Embryologia, v.37, p.317-321, 2008. Disponível em: <http://www3.interscience.wiley.com/journal/120122341/ abstract?CRETRY=1\&SRETRY=0>. Acessso em: 30 mai. 2009. doi: 10.1111/j.1439-0264.2008.00855.x.

PINTO, M.R.A. et al. Os arranjos configurados pelas artérias mesentéricas cranial e caudal no pato doméstico (Cairina moshata). Brazilian Journal of Veterinary Research and Animal Science, v.35, p.107-109, 1998. Disponível em: <http:/ /www.scielo.br/scielo.php?script=sci_arttext\&pid=S141395961998000300002\&Ing=pt\&nrm=iso $>$. Acesso em: 30 mai. 2009. doi: 10.1590/S1413-95961998000300002.

RODRIGUES, R.F. et al. Vascularização arterial do trato gastrointestinal da Trachemys scripta elegans, Wied, 1838. Brazilian Journal of Veterinary Research and Animal Science, v.40, p.63-68, 2003. Disponível em: <http:// www.scielo.br/pdf/bjvras/v40n1/19296.pdf >. Acesso em: 30 mai. 2009. ISSN: 1678-4456 (Online).

SCHWARZE, E.; SCHRÖDER L. Compendio de anatomia veterinária. Tomo II: el sistema visceral. Zaragoza: Acribia, 1970. 313p

SIQUEIRA, S.L.; LÁZARO-DA-SILVA, A. Contribuição à anatomia arterial do cólon sigmóide aplicável a operações de abaixamento. Arquivos de Gastroenterologia, v.40, n.4, p.209-215, 2003. Disponível em: <http://www.scielo.br/scielo.php?pid=S000428032003000400003\&script=sci_arttext $>$. Acesso em: 30 mai. 2009. doi: 10.1590/S0004-28032003000400003.

STACCHINI, A. et al. Artificial sphincters as surgical treatment for experimental massive resection of small intestine. American Journal of Surgery, v.143, p.721-726, 1982.

TESTUT, L.; LATARJET A. Tratado de anatomia humana. Buenos Aires: Salvat, 1960. Tomo IV, 1168p.

ZAPPALÁ, A.A. Base anatômica da ressecção segmentar do baço. Anais da Faculdade de Medicina da Universidade do Recife, v.23, p.7-36, 1963. 